\title{
ON QUASI-ORTHODOX SEMIGROUPS WITH INVERSE TRANSVERSALS
}

\author{
by T. S. BLYTH and M. H. ALMEIDA SANTOS*
}

(Received 20th September 1995)

\begin{abstract}
An inverse transversal of a regular semigroup $S$ is an inverse subsemigroup $S^{\circ}$ that contains precisely one inverse of each element of $S$. Here we consider the case where $S$ is quasi-orthodox. We give natural characterisations of such semigroups and consider various properties of congruences.
\end{abstract}

1991 Mathematics subject classification: $20 \mathrm{M} 17$.

An inverse transversal of a regular semigroup $S$ is an inverse subsemigroup $T$ with the property that $|T \cap V(x)|=1$ for every $x \in S$, where $V(x)$ denotes the set of inverses of $x \in S$. In what follows we shall write the unique element of $T \cap V(x)$ as $x^{\circ}$, and $T$ as $S^{\circ}=\left\{x^{\circ} ; x \in S\right\}$. Then in $S^{\circ}$ we have $\left(x^{\circ}\right)^{-1}=x^{\circ \circ}$, so that $x^{\circ}=x^{000}$ for every $x \in S$. Fundamental properties of the unary operation $x \mapsto x^{\circ}$ in such a semigroup are

(a) [4] $(\forall x, y \in S) \quad(x y)^{\circ}=\left(x^{\circ} x y\right)^{\circ} x^{\circ}=y^{\circ}\left(x y y^{\circ}\right)^{\circ}=y^{\circ}\left(x^{\circ} x y y^{\circ}\right)^{\circ} x^{\circ}$;

(B) [2] $(\forall x, y \in S) \quad\left(x y^{\circ}\right)^{\circ}=y^{\circ \circ} x^{\circ}, \quad\left(x^{\circ} y\right)^{\circ}=y^{\circ} x^{\circ \circ}$;

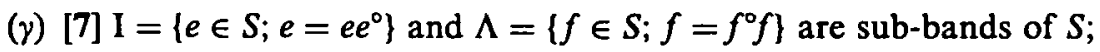

(ס) [5] $S$ is orthodox if and only if $(\forall x, y \in S) \quad(x y)^{\circ}=y^{\circ} x^{\circ}$.

The sub-bands I and $\Lambda$, which are respectively left regular and right regular, are such that $I \cap \Lambda$ is the semilattice $E\left(S^{\circ}\right)$ of idempotents of $S^{\circ}$. Together with the inverse subsemigroup $S^{\circ}$, they form the building bricks in the structure theorems of Saito [5].

In this paper we shall be concerned primarily with the case where $S$ is quasiorthodox. Yamada [8] has defined a semigroup $S$ to be quasi-orthodox if there is an inverse semigroup $\Gamma$ and a surjective morphism $\varphi: S \rightarrow \Gamma$ such that, for every idempotent $e \in \Gamma$, the pre-image of $e$ under $\varphi$ is a completely simple subsemigroup of $S$. A regular semigroup $S$ is quasi-orthodox if and only if the subsemigroup $\langle E(S)\rangle$ is completely regular.

Saito [6] has proved that if $S$ is regular with an inverse transversal $S^{\circ}$ then the following statements are equivalent:

\footnotetext{
* The second-named author gratefully acknowledges support from INVOTAN, and Research Grant PBIC/ C/MAT/2141/96 from NICT (Portugal).
} 
(1) $S$ is quasi-orthodox;

(2) $(\forall x, y \in S) \quad(x y)^{\circ}(x y)^{\circ 0}=y^{\circ} x^{\circ} x^{\circ 0} y^{\circ 0}$;

(3) $(\forall x, y \in S) \quad(x y)^{\circ \circ}(x y)^{\circ}=x^{\circ \circ} y^{\circ \circ} y^{\circ} x^{\circ}$.

Since Green's relations $\mathcal{L}$ and $\mathcal{R}$ on $S$ are given by

$$
(x, y) \in \mathcal{L} \Leftrightarrow x^{\circ} x=y^{\circ} y, \quad(x, y) \in \mathcal{R} \Leftrightarrow x x^{\circ}=y y^{\circ},
$$

it follows immediately that

(e) $S$ is quasi-orthodox if and only if

$$
(\forall x, y \in S) \quad\left((x y)^{\circ}, y^{\circ} x^{\circ}\right) \in \mathcal{H} .
$$

In the same paper, Saito proved that if $S$ is quasi-orthodox then $S$ is orthodox if and only if $S^{\circ}$ is weakly multiplicative, in the sense that $(\Lambda \mathrm{I})^{\circ} \subseteq E\left(S^{\circ}\right)$. Now if $S$ is orthodox it follows by $(\delta)$ and $(\epsilon)$ that $S$ is quasi-orthodox. Since $i^{\circ} \in E\left(S^{\circ}\right)$ for every $i \in I$ and $l^{\circ} \in E\left(S^{\circ}\right)$ for every $l \in \Lambda$, it is clear that if $S$ is orthodox then $S^{\circ}$ is weakly multiplicative. Hence the following statements are equivalent:

(1) $S$ is orthodox;

(2) $S$ is quasi-orthodox and $S^{\circ}$ is weakly multiplicative.

Example 1. Let $S$ be the set of real singular $2 \times 2$ matrices having a non-zero entry in the $(1,1)$-position, and let $M$ consist of $S$ with the $2 \times 2$ zero matrix adjoined. Then, as we have shown in [1], $M$ is a regular semigroup and relative to the definitions

$$
\left[\begin{array}{cc}
a & b \\
c & a^{-1} b c
\end{array}\right]^{\circ}=\left[\begin{array}{cc}
a^{-1} & 0 \\
0 & 0
\end{array}\right], \quad\left[\begin{array}{ll}
0 & 0 \\
0 & 0
\end{array}\right]^{\circ}=\left[\begin{array}{ll}
0 & 0 \\
0 & 0
\end{array}\right]
$$

the set

$$
M^{\circ}=\left\{\left[\begin{array}{ll}
x & 0 \\
0 & 0
\end{array}\right] ; x \neq 0\right\} \cup\left\{\left[\begin{array}{ll}
0 & 0 \\
0 & 0
\end{array}\right]\right\}
$$

is an inverse transversal of $M$.

Consider the subset $Q$ of $M$ given by

$$
Q=\left\{\left[\begin{array}{ll}
x & x \\
x & x
\end{array}\right],\left[\begin{array}{ll}
x & 0 \\
x & 0
\end{array}\right],\left[\begin{array}{cc}
x & x \\
0 & 0
\end{array}\right],\left[\begin{array}{cc}
x & 0 \\
0 & 0
\end{array}\right] ; x \neq 0\right\} .
$$

It is readily seen that $Q$ is a subsemigroup of $M$. Since $Q$ is clearly closed under the operation $A \mapsto A^{\circ}$ we have that $Q$ is regular with

$$
Q^{\circ}=\left\{\left[\begin{array}{ll}
x & 0 \\
0 & 0
\end{array}\right] ; x \neq 0\right\}
$$


an inverse (in fact, a group) transversal of $Q$. Since $E\left(Q^{\circ}\right)$ is a singleton and since each of $(A B)^{\circ}(A B)^{\circ \circ}$ and $B^{\circ} A^{\circ} A^{\circ \circ} B^{\circ \circ}$ belong to $E\left(Q^{\circ}\right)$, it follows that $(A B)^{\circ}(A B)^{\circ \circ}=B^{\circ} A^{\circ} A^{\circ \circ} B^{\circ 0}$ and therefore $Q$ is quasi-orthodox. That $Q$ is not orthodox can be seen from the observation that $\left[\begin{array}{ll}1 & 1 \\ 0 & 0\end{array}\right]$ and $\left[\begin{array}{ll}1 & 0 \\ 1 & 0\end{array}\right]$ belong to $E(Q)$ but

$$
\left[\begin{array}{ll}
1 & 1 \\
0 & 0
\end{array}\right]\left[\begin{array}{ll}
1 & 0 \\
1 & 0
\end{array}\right]=\left[\begin{array}{ll}
2 & 0 \\
0 & 0
\end{array}\right] \notin E(Q) .
$$

In what follows we shall require certain properties of congruences. For this purpose, we let $\operatorname{Con} S$ be the lattice of congruences on the semigroup $S$, and denote by $\overline{\operatorname{Con}} S$ the sublattice of ${ }^{\circ}$-congruence, i.e., congruences $\vartheta$ with the property that $(x, y) \in \vartheta$ implies $\left(x^{\circ}, y^{\circ}\right) \in \vartheta$. In $\left[2\right.$, Theorem 1] we have shown that if $X \in\left\{I, S^{\circ}, \Lambda\right\}$ then Con $X=\overline{\operatorname{Con}} X$.

Of particular interest are those congruences on $X \in\left\{I, S^{\circ}, \Lambda\right\}$ that are special in the sense that they can be extended to ${ }^{\circ}$-congruences on $S$. In [2, Theorem 8] we have shown that $\imath \in$ Con $I$ is special if and only if

$$
(i, j) \in \imath \Rightarrow(\forall x \in S) \quad\left(x i(x i)^{\circ}, x j(x j)^{\circ}\right) \in \imath .
$$

In what follows, for $X \in\left\{I, S^{\circ}, \Lambda\right\}$ we shall denote Green's relations on $X$ by $\mathcal{L}_{X}$, $\mathcal{R}_{X}$, and $\mathcal{H}_{X}$. By a result of Hall [3] we have that $\mathcal{L}_{X}, \mathcal{R}_{X}, \mathcal{H}_{X}$ are respectively the restrictions to $X$ of $\mathcal{L}, \mathcal{R}, \mathcal{H}$ on $S$.

Theorem 1. If $S$ is a regular semigroup with an inverse transversal $S^{\circ}$ then $\mathcal{L}_{\mathrm{I}} \in \operatorname{Con} \mathrm{I}$ and

$$
(i, j) \in \mathcal{L}_{1} \Leftrightarrow i^{\circ}=j^{\circ} .
$$

Moreover, $\mathcal{R}_{\mathrm{I}}$ reduces to equality.

Proof. Since $I$ is left regular, $\mathcal{L}_{\mathrm{I}}$ is a congruence and $\mathcal{R}_{\mathrm{I}}$ reduces to equality. Now, since I is orthodox with $E\left(S^{\circ}\right)$ a semilattice transversal, we have

$$
(i, j) \in \mathcal{L}_{\mathrm{I}} \Rightarrow i^{\circ}=(i j)^{\circ}=j^{\circ} i^{\circ}=i^{\circ} j^{\circ}=(j i)^{\circ}=j^{\circ} .
$$

Conversely, let $i, j \in \mathrm{I}$ be such that $i^{\circ}=j^{\circ}$. Since $i=i^{\circ}$ and $i^{\circ} i=i^{\circ}$ we have $\left(i, i^{\circ}\right) \in \mathcal{L}_{\mathrm{l}}$. Consequently, $i^{\circ}=j^{\circ}$ implies that $(i, j) \in \mathcal{L}_{1}$.

Corollary. $\mathcal{L}_{\mathrm{I}}$ is special if and only if, for all $i, j \in \mathrm{I}$,

$$
i^{\circ}=j^{\circ} \Rightarrow(\forall x \in S) \quad(x i)^{\circ \circ}(x i)^{\circ}=(x j)^{\circ \circ}(x j)^{\circ} .
$$

We shall denote by $\mu$ the biggest idempotent-separating congruence on $S^{\circ}$. For every idempotent-separating congruence $\pi$ on $S^{\circ}$ we let $\Theta_{\pi}$ be the relation defined on $S$ by

$$
(a, b) \in \Theta_{\pi} \Leftrightarrow\left(a^{\circ}, b^{\circ}\right) \in \pi .
$$


Theorem 2. If $S$ is a regular semigroup with an inverse transversal $S^{\circ}$ then the following statements are equivalent:

(1) $S$ is quasi-orthodox;

(2) $\mathcal{L}_{\mathrm{I}}$ is special;

(3) $\mathcal{R}_{\Lambda}$ is special;

(4) $\Theta_{\mu} \in \overline{\operatorname{Con}} S$.

Proof. (1) $\Rightarrow$ (2): If (1) holds then

$$
(x i)^{\circ \circ}(x i)^{\circ}=x^{\circ \circ} i^{\circ \circ} i^{\circ} x^{\circ}=x^{\circ \circ} i^{\circ} x^{\circ} .
$$

Then (2) follows by the Corollary to Theorem 1.

$(2) \Rightarrow(1)$ : For every $y \in S$, we have $\left(y y^{\circ}\right)^{\circ}=y^{\circ \circ} y^{\circ}=\left(y^{\circ \circ} y^{\circ}\right)^{\circ}$. If (2) holds, then it follows by the Corollary to Theorem 1 that

$$
(\forall x, y \in S) \quad\left(x y y^{\circ}\right)^{\circ \circ}\left(x y y^{\circ}\right)^{\circ}=\left(x y^{\circ \circ} y^{\circ}\right)^{\circ \circ}\left(x y^{\circ \circ} y^{\circ}\right)^{\circ} .
$$

Now, on the one hand,

$$
\begin{aligned}
\left(x y y^{\circ}\right)^{\circ \circ}\left(x y y^{\circ}\right)^{\circ} & =\left(x y y^{\circ}\right)^{\circ \circ}\left(y y^{\circ}\right)^{\circ}\left(x y y^{\circ}\left(y y^{\circ}\right)^{\circ}\right)^{\circ} \\
& =\left(x y y^{\circ}\right)^{\circ \circ} y^{\circ \circ} y^{\circ}\left(x y y^{\circ}\right)^{\circ} \\
& =\left(y^{\circ}\left(x y y^{\circ}\right)^{\circ}\right)^{\circ} y^{\circ}\left(x y y^{\circ}\right)^{\circ} \\
& =(x y)^{\circ \circ}(x y)^{\circ} ;
\end{aligned}
$$

and, on the other hand,

$$
\left(x y^{\circ \circ} y^{\circ}\right)^{\circ \circ}\left(x y^{\circ \circ} y^{\circ}\right)^{\circ}=x^{\circ \circ} y^{\circ \circ} y^{\circ} y^{\circ \circ} y^{\circ} x^{\circ}=x^{\circ \circ} y^{\circ \circ} y^{\circ} x^{\circ} \text {. }
$$

Thus $(x y)^{\circ \circ}(x y)^{\circ}=x^{\circ \circ} y^{\circ \circ} y^{\circ} x^{\circ}$ and so $S$ is quasi-orthodox.

(1) $\Leftrightarrow(3)$ : This is similar.

(1) $\Rightarrow$ (4): Suppose that (1) holds and that $(a, b) \in \Theta_{\mu}$. Then for every $e \in E\left(S^{\circ}\right)$ we have $a^{\circ} e a^{\circ \circ}=b^{\circ} e b^{\circ \circ}$. Since $e=e^{\circ}=e^{\circ \circ}$ for every $e \in E\left(S^{\circ}\right)$, it follows that, for every $x \in S$,

$$
\begin{aligned}
(a x)^{\circ} e(a x)^{\circ \circ} & =(a x)^{\circ} e^{\circ} e^{\circ \circ}(a x)^{\circ \circ} \\
& =(e a x)^{\circ}(e a x)^{\circ 0} \\
& =x^{\circ}(e a)^{\circ}(e a)^{\circ \circ} x^{\circ \circ} \text { by (1) } \\
& =x^{\circ} a^{\circ} e a^{\circ \circ} x^{\circ \circ} \\
& =x^{\circ} b^{\circ} e b^{\circ \circ} x^{\circ \circ} \\
& =(b x)^{\circ} e(b x)^{\circ \circ} .
\end{aligned}
$$


Also,

$$
\begin{aligned}
(x a)^{\circ} e(x a)^{\circ \circ} & =(e x a)^{\circ}(e x a)^{\circ 0} & \\
& =a^{\circ}(e x)^{\circ}(e x)^{\circ \circ} a^{\circ \circ} & \text { by }(1) \\
& =b^{\circ}(e x)^{\circ}(e x)^{\circ \circ} b^{\circ \circ} & \text { since }(e x)^{\circ}(e x)^{\circ \circ} \in E\left(S^{\circ}\right) \\
& =(x b)^{\circ} e(x b)^{\circ \circ} . &
\end{aligned}
$$

Consequently, $\Theta_{\mu} \in$ Con $S$. Clearly, if $(a, b) \in \Theta_{\mu}$ then $\left(a^{\circ}, b^{\circ}\right) \in \Theta_{\mu}$. Hence $\Theta_{\mu} \in \overline{\operatorname{Con}} S$.

(4) $\Rightarrow$ (1): If $\Theta_{\mu} \in \overline{\operatorname{Con}} S$ then, observing that $\left(x, x^{\circ \circ}\right) \in \Theta_{\mu}$ for every $x \in S$, we have that $\left(x y, x^{\circ \circ} y^{\circ \circ}\right) \in \Theta_{\mu}$ for all $x, y \in S$ and therefore

$$
(x y)^{\circ} x^{\circ 0} x^{\circ}(x y)^{\circ 0}=\left(x^{00} y^{\circ 0}\right)^{\circ} x^{\circ 0} x^{\circ}\left(x^{\circ 0} y^{\circ 0}\right)^{\circ 0} \text {. }
$$

Clearly, the right hand side reduces to $y^{\circ} x^{\circ} x^{\circ \circ} y^{\circ \circ}$. As for the left hand side, this can be written $\left(x^{\circ} x y\right)^{\circ} x^{\circ}(x y)^{\circ \circ}=(x y)^{\circ}(x y)^{\circ \circ}$. Hence we see that $S$ is quasi-orthodox.

Theorem 3. Let $S$ be a regular semigroup with an inverse transversal $S^{\circ}$ and let $\pi$ be an idempotent-separating congruence on $S^{\circ}$. Then the following statements are equivalent:

(1) $\Theta_{\pi} \in \overline{\operatorname{Con}} S$;

(2) $(\forall x, y \in S) \quad\left((x y)^{\circ}, y^{\circ} x^{\circ}\right) \in \pi$;

(3) $(\forall i \in \mathrm{I})(\forall l \in \Lambda) \quad\left((l i)^{\circ}, l^{\circ} i^{\circ}\right) \in \pi$.

Proof. (1) $\Rightarrow$ (2): Clearly, for every $x \in S$, we have $\left(x, x^{\circ 0}\right) \in \Theta_{\pi}$ so if (1) holds we have $\left(x y, x^{\circ \circ} y^{\circ \circ}\right) \in \Theta_{\pi}$ whence $\left((x y)^{\circ}, y^{\circ} x^{\circ}\right) \in \pi$.

(2) $\Rightarrow(3)$ : This is clear.

(3) $\Rightarrow$ (1): If (3) holds, we observe that, for $i, j \in \mathrm{I}$ and $l, m \in \Lambda$,

$$
i^{\circ}=j^{\circ}, l^{\circ}=m^{\circ} \Rightarrow\left((l i)^{\circ},(m j)^{\circ}\right) \in \pi .
$$

Hence, if $(x, y) \in \Theta_{\pi}$ we have $\left(x^{\circ} x^{\circ \circ}, y^{\circ} y^{\circ \circ}\right) \in \pi$ and therefore, since $\pi$ is idempotentseparating, $x^{\circ} x^{\circ \circ}=y^{\circ} y^{\circ 0}$. Applying the above observation we deduce that, for every $a \in S$,

$$
\left(\left(x^{\circ} x a a^{\circ}\right)^{\circ},\left(y^{\circ} y a a^{\circ}\right)^{\circ}\right) \in \pi .
$$

Since $\left(x^{\circ}, y^{\circ}\right) \in \pi$ it follows that

$$
\left((x a)^{\circ},(y a)^{\circ}\right)=\left(a^{\circ}\left(x^{\circ} x a a^{\circ}\right)^{\circ} x^{\circ}, a^{\circ}\left(y^{\circ} y a a^{\circ}\right)^{\circ} y^{\circ}\right) \in \pi
$$


and therefore $(x a, y a) \in \Theta_{\pi}$. Similarly, we can show that $(a x, a y) \in \Theta_{\pi}$, and therefore $\Theta_{\pi} \in \operatorname{Con} S$. It now follows from the definition of $\Theta_{\pi}$ that $\Theta_{\pi} \in \overline{\operatorname{Con}} S$.

Corollary. S is quasi-orthodox if and only if $\mu$ is such that

$$
(\forall x, y \in S) \quad\left((x y)^{\circ}, y^{\circ} x^{\circ}\right) \in \mu .
$$

Proof. This follows immediately by Theorem 2 .

In order to proceed, we require some general facts concerning ${ }^{\circ}$-congruences. In [2] we have established the general form of such congruences. Specifically, we define a triple $(l, \pi, \lambda) \in \operatorname{Con} I \times \overline{\operatorname{Con}} S^{\circ} \times \operatorname{Con} \Lambda$ to be

(a) balanced if $\left.\imath\right|_{E\left(S^{\circ}\right)}=\left.\pi\right|_{E\left(S^{\circ}\right)}=\left.\lambda\right|_{E\left(S^{\circ}\right)}$;

(b) linked if for all $i_{1}, i_{2} \in \mathbf{I}$, all $x_{1}, x_{2} \in S^{\circ}$, and all $l_{1}, l_{2} \in \Lambda$,

$$
\begin{aligned}
& \left(i_{1}, i_{2}\right) \in \imath,\left(l_{1}, l_{2}\right) \in \lambda \Rightarrow \begin{cases}\left(l_{1} i_{1}\left(l_{1} i_{1}\right)^{\circ}, l_{2} i_{2}\left(l_{2} i_{2}\right)^{\circ}\right) \in \iota & {[\alpha]} \\
\left(\left(l_{1} i_{1}\right)^{\circ},\left(l_{2} i_{2}\right)^{\circ}\right) \in \pi & {[\beta]} \\
\left(\left(l_{1} i_{1}\right)^{\circ} l_{1} i_{1},\left(l_{2} i_{2}\right)^{\circ} l_{2} i_{2}\right) \in \lambda & {[\gamma]}\end{cases} \\
& \left(i_{1}, i_{2}\right) \in \imath,\left(x_{1}, x_{2}\right) \in \pi \Rightarrow\left(x_{1} i_{1} x_{1}^{\circ}, x_{2} i_{2} x_{2}^{0}\right) \in l \quad[\delta] \\
& \left(l_{1}, l_{2}\right) \in \lambda,\left(x_{1}, x_{2}\right) \in \pi \Rightarrow\left(x_{1}^{\circ} l_{1} x_{1}, x_{2}^{\circ} l_{2} x_{2}\right) \in \lambda \quad[\epsilon]
\end{aligned}
$$

The set BLT(S) of balanced linked triples forms a lattice that is isomorphic to $\overline{\operatorname{Con}} S$. Every $\vartheta \in \overline{\operatorname{Con}} S$ is of the form $\Psi(l, \pi, \lambda)$ where $(l, \pi, \lambda) \in \operatorname{BLT}(S)$ and

$$
(a, b) \in \Psi(l, \pi, \lambda) \Leftrightarrow\left(a a^{\circ}, b b^{\circ}\right) \in \iota, \quad\left(a^{\circ}, b^{\circ}\right) \in \pi, \quad\left(a^{\circ} a, b^{\circ} b\right) \in \lambda .
$$

Concerning the special congruences on $X \in\left\{I, S^{\circ}, \Lambda\right\}$ we have also established in [2] the following results:

(A) if $\imath \in \mathrm{Con} I$ is special then the biggest extension of $\imath$ to a ${ }^{\circ}$-congruence on $S$ is the congruence $\hat{\imath} \in \overline{\mathrm{Con}} S$ given by

$$
(a, b) \in \hat{\imath} \Leftrightarrow(\forall i \in \mathrm{I}) \quad\left(a i(a i)^{\circ}, b i(b i)^{\circ}\right) \in \imath ;
$$

(B) a dual result if $\lambda \in \operatorname{Con} \Lambda$ is special;

(C) $\pi \in \operatorname{Con} S^{\circ}$ is special if and only if

$$
(x, y) \in \pi \Rightarrow(\forall i \in I)(\forall l \in \Lambda) \quad\left((l x i)^{\circ},(l y i)^{\circ}\right) \in \pi .
$$

In this case the biggest extension of $\pi \in \operatorname{Con} S^{\circ}$ to a ${ }^{\circ}$-congruence on $S$ is the congruence $\hat{\pi} \in \overline{\mathrm{Con}} S$ given by

$$
(a, b) \in \hat{\pi} \Leftrightarrow(\forall i \in \mathrm{I})(\forall l \in \Lambda) \quad\left((l a i)^{\circ},(l b i)^{\circ}\right) \in \pi .
$$


In view of Theorem 2 , when $S$ is quasi-orthodox there are balanced linked triples of the form $\left(\mathcal{L}_{1},-,-\right)$ and $\left(-,-, \mathcal{R}_{\Lambda}\right)$. Since, by Theorem $1, \mathcal{L}$ and $\mathcal{R}$ reduce to equality on $E\left(S^{\circ}\right)$, the middle components of any such triples must be idempotent-separating congruences on $S^{\circ}$. Conversely, any balanced linked triple of the form $(1, \pi, \lambda)$ in which $\pi$ is idempotent-separating is such that $\iota \subseteq \mathcal{L}_{\mathrm{I}}$ and $\lambda \subseteq \mathcal{R}_{\Lambda}$. In fact, since in such a triple $\left.l\right|_{E(S)}$ is equality we have, by Theorem 1 ,

$$
\left(i_{1}, i_{2}\right) \in \imath \Rightarrow i_{1}^{\circ}=i_{2}^{\circ} \Rightarrow\left(i_{1}, i_{2}\right) \in \mathcal{L}_{1},
$$

so that $\imath \subseteq \mathcal{L}_{\mathrm{I}}$, and similarly $\lambda \subseteq \mathcal{R}_{\Lambda}$.

In order to establish the existence of balanced linked triples of the form $\left(\mathcal{L}_{\mathrm{I}},-, \mathcal{R}_{\Lambda}\right)$ or, equivalently, the existence of ${ }^{\circ}$-congruences on $S$ that simultaneously extend $\mathcal{L}_{\mathrm{I}}$ and $\mathcal{R}_{\Lambda}$, we require the following general result.

Theorem 4. Let $S$ be a regular semigroup with an inverse transversal $S^{\circ}$. Given special congruences $1 \in$ Con I and $\lambda \in$ Con $\Lambda$, there exists a balanced linked triple of the form $(l,-, \lambda)$ if and only if $\left.\imath \subseteq \hat{\lambda}\right|_{\mathrm{I}}$ and $\left.\lambda \subseteq \hat{\imath}\right|_{\Lambda}$. In this case, the biggest such triple has middle component $\left.\left.\hat{\imath}\right|_{s^{\circ}} \cap \hat{\lambda}\right|_{s^{\circ}}$.

Proof. Suppose that $\imath \in \operatorname{Con} I$ and $\lambda \in \operatorname{Con} \Lambda$ are such that there is a balanced linked triple of the form $(l,-, \lambda)$. Observe that, by $[\alpha]$, if $\left(l_{1}, l_{2}\right) \in \lambda$ then for every $i \in I$, $\left(l_{1} i\left(l_{1} i\right)^{\circ}, l_{2} i\left(l_{2} i\right)^{\circ}\right) \in \imath$ and therefore $\left.\left(l_{1}, l_{2}\right) \in \hat{i}\right|_{\Lambda}$. Thus $\left.\lambda \subseteq \hat{\imath}\right|_{\Lambda}$, and similarly $\left.\imath \subseteq \hat{\lambda}\right|_{\mathrm{I}}$.

Conversely, $\hat{i}$ corresponds to the balanced linked triple $\left(l,\left.\hat{l}\right|_{S^{\circ}},\left.\hat{\imath}\right|_{\Lambda}\right)$, and $\hat{\lambda}$ corresponds to the balanced linked triple $\left(\left.\hat{\lambda}\right|_{I},\left.\hat{\lambda}\right|_{s^{s}}, \lambda\right)$. Therefore, if the stated conditions hold, $i \cap \hat{\lambda}$ corresponds to the balanced linked triple $\left(l,\left.\left.\hat{i}\right|_{s} \cap \hat{\lambda}\right|_{s}, \lambda\right)$.

Finally, for any balanced linked triple $(l, \pi, \lambda)$, it follows by $[\delta]$ that, for $x_{1}, x_{2} \in S^{\circ}$,

$$
\begin{aligned}
\left(x_{1}, x_{2}\right) \in \pi & \Rightarrow(\forall i \in \mathrm{I}) \quad\left(x_{1} i\left(x_{1} i\right)^{\circ}, x_{2} i\left(x_{2} i\right)^{\circ}\right)=\left(x_{1} i x_{1}^{\circ}, x_{2} i x_{2}^{\circ}\right) \in \imath \\
& \left.\Rightarrow\left(x_{1}, x_{2}\right) \in \hat{\imath}\right|_{S^{\circ}}
\end{aligned}
$$

and so $\left.\pi \subseteq \hat{i}\right|_{s^{\circ}}$. Similarly, $\left.\pi \subseteq \hat{\lambda}\right|_{s^{\circ}}$ and we conclude that the biggest balanced linked triple of the form $(l,-, \lambda)$ has $\left.\left.\hat{\imath}\right|_{S^{\circ}} \cap \hat{\lambda}\right|_{S^{\circ}}$ as its middle component.

To see that, when $S$ is quasi-orthodox, there exist balanced linked triples of the form $\left(\mathcal{L}_{1},-, \mathcal{R}_{\Lambda}\right)$ we may use Theorem 4 as follows. By Theorem 2 we can consider $\widehat{\mathcal{L}}_{\mathrm{I}}$ and $\widehat{\mathcal{R}_{\Lambda}}$. Now, by $(A)$ and Theorem 1 , we have

$$
\begin{aligned}
& (a, b) \in \widehat{\mathcal{L}}_{\mathrm{I}} \Leftrightarrow(\forall i \in \mathrm{I}) \quad\left(a i(a i)^{\circ}, b i(b i)^{\circ}\right) \in \mathcal{L}_{\mathrm{I}} \\
& \Leftrightarrow(\forall i \in \mathrm{I}) \quad(a i)^{\circ \circ}(a i)^{\circ}=(b i)^{\circ \circ}(b i)^{\circ} \\
& \Leftrightarrow(\forall i \in \mathrm{I}) \quad a^{\circ \circ} i^{\circ} a^{\circ}=b^{\circ \circ} i^{\circ} b^{\circ} \text {, }
\end{aligned}
$$

and similarly,

$$
(a, b) \in \widehat{\mathcal{R}_{\Lambda}} \Leftrightarrow(\forall l \in \Lambda) \quad a^{\circ} l^{\circ} a^{\circ \circ}=b^{\circ} l^{\circ} b^{\circ \circ}
$$


Since $E\left(S^{\circ}\right)=\left\{i^{\circ} ; i \in \mathrm{I}\right\}=\left\{l^{\circ} ; l \in \Lambda\right\}$, we deduce from these expressions that

$$
(a, b) \in \widehat{\mathcal{L}_{1}} \Leftrightarrow(a, b) \in \widehat{\mathcal{R}_{\Lambda}} .
$$

It follows that $\widehat{\mathcal{L}}_{1}=\widehat{\mathcal{R}}_{\Lambda}$. Consequently $\mathcal{L}_{1}=\left.\widehat{\mathcal{L}}_{1}\right|_{1}=\left.\widehat{\mathcal{R}}_{\Lambda}\right|_{\mathrm{I}}$ and similarly $\mathcal{R}_{\Lambda}=\left.\widehat{\mathcal{L}}_{\mathrm{I}}\right|_{\Lambda}$. Thus, by Theorem 4 , balanced linked triples of the form $\left(\mathcal{L}_{1},-, \mathcal{R}_{\Lambda}\right)$, and hence ${ }^{\circ}$-congruences on $S$ that simultaneously extend $\mathcal{L}_{\mathrm{I}}$ and $\mathcal{R}_{\Lambda}$, exist.

We now determine precisely the nature of middle components in such triples.

Theorem 5. Let $S$ be a quasi-orthodox semigroup with an inverse transversal $S^{\circ}$. Then $\left(\mathcal{L}_{1}, \pi, \mathcal{R}_{\mathrm{\Lambda}}\right) \in \operatorname{BLT}(S)$ if and only if $\pi$ is idempotent-separating on $S^{\circ}$ and $\Theta_{\pi} \in \overline{\operatorname{Con}} S$.

Proof. $\Rightarrow$ : If $\left(\mathcal{L}_{\mathrm{I}}, \pi, \mathcal{R}_{\Lambda}\right) \in \operatorname{BLT}(S)$ then, by the above observations, $\pi$ is idempotent-separating and $\left(\mathcal{L}_{\mathrm{I}}, \pi, \mathcal{R}_{\Lambda}\right)$ is the biggest balanced linked triple of the form $(-, \pi,-)$. Consequently, $\hat{\pi}=\Psi\left(\mathcal{L}_{1}, \pi, \mathcal{R}_{\Lambda}\right)$ and therefore

$$
\begin{aligned}
(x, y) \in \hat{\pi} & \Leftrightarrow x^{\circ} x^{\circ \circ}=y^{\circ} y^{\circ \circ},\left(x^{\circ}, y^{\circ}\right) \in \pi, x^{\circ \circ} x^{\circ}=y^{\circ \circ} y^{\circ} \\
& \Leftrightarrow\left(x^{\circ}, y^{\circ}\right) \in \pi
\end{aligned}
$$

and so $\hat{\pi}=\Theta_{\pi}$. Hence $\Theta_{\pi} \in \overline{\operatorname{Con}} S$.

$\Leftarrow$ : If $\pi$ is idempotent-separating on $S^{\circ}$ and $\Theta_{\pi} \in \overline{\operatorname{Con}} S$ then it is readily seen that $\left.\Theta_{\pi}\right|_{\mathrm{I}}=\mathcal{L}_{\mathrm{I}},\left.\Theta_{\pi}\right|_{S^{\circ}}=\pi$, and $\left.\Theta_{\pi}\right|_{\Lambda}=\mathcal{R}_{\Lambda}$. Consequently $\Theta_{\pi}=\Psi\left(\mathcal{L}_{\mathrm{l}}, \pi, \mathcal{R}_{\Lambda}\right)$.

Corollary. Let $S$ be a quasi-orthodox semigroup with an inverse transversal $S^{\circ}$. Then

(1) the biggest idempotent-separating congruence $\mu$ on $S^{\circ}$ is special with $\left(\mathcal{L}_{1}, \mu, \mathcal{R}_{\Lambda}\right)$ the biggest balanced linked triple of the form $\left(\mathcal{L}_{\mathrm{I}},-, \mathcal{R}_{\Lambda}\right)$;

(2) the biggest extension of $\mu$ in $\overline{\operatorname{Con}} S$ is $\Psi\left(\mathcal{L}_{\mathrm{I}}, \mu, \mathcal{R}_{\mathrm{\Lambda}}\right)=\Theta_{\mu}$.

Proof. This follows from Theorems 2,4 and 5 on observing that $\left.\widehat{\mathcal{L}_{\mathrm{I}}}\right|_{s^{\circ}}=\left.\widehat{\mathcal{R}_{\Lambda}}\right|_{s^{\circ}}=\mu$.

In what follows we shall denote by $\zeta$ the ${ }^{\circ}$-congruence on $S$ generated by the set $\left\{\left(a, a^{\circ)}\right) ; a \in S\right\}$.

Theorem 6. Let $S$ be a quasi-orthodox semigroup with an inverse transversal $S^{\circ}$. Then the smallest balanced linked triple of the form $\left(\mathcal{L}_{1},-, \mathcal{R}_{\Lambda}\right)$ has middle component $\left.\zeta\right|_{s^{\circ}}$.

Proof. Let $T$ be the set of idempotent-separating congruences $\pi$ on $S^{\circ}$ such that $\Theta_{\pi} \in \overline{\operatorname{Con}} S$. Then for every $a \in S$ we have $\left(a, a^{\circ \circ}\right) \in \bigcap_{\pi \in T} \Theta_{\pi}$. It follows that $\zeta \subseteq \bigcap_{\pi \in T} \Theta_{\pi}$. For every $\pi \in T$ we then have $\zeta \subseteq \Theta_{\pi}$ and so $\left.\left.\zeta\right|_{s} \subseteq \Theta_{\pi}\right|_{S^{\circ}}=\pi$. Hence $\left.\zeta\right|_{S^{\circ}}$ is idempotentseparating. Now

$$
(a, b) \in \zeta \Leftrightarrow\left(a^{\circ \circ}, b^{\circ \circ}\right) \in \zeta \Leftrightarrow\left(a^{\circ}, b^{\circ}\right) \in \zeta \Leftrightarrow(a, b) \in \Theta_{\zeta \mid s} .
$$


Consequently, $\zeta=\Theta_{\mid l_{s}}$ and therefore $\left.\zeta\right|_{s} \in T$. It follows that $\left.\zeta\right|_{s^{\circ}}=\min T$ whence we obtain the result by Theorem 5 .

We can now describe the balanced linked triples of the form $\left(\mathcal{L}_{1},-, \mathcal{R}_{\Lambda}\right)$.

Theorem 7. Let $S$ be a quasi-orthodox semigroup with an inverse transversal $S^{\circ}$. Then $\left(\mathcal{L}_{1}, \pi, \mathcal{R}_{\Lambda}\right) \in \operatorname{BLT}(S)$ if and only if $\pi$ belongs to the interval $\left[\left.\zeta\right|_{s^{\circ}}, \mu\right]$ of Con $S^{\circ}$.

Proof. $\Rightarrow$ : If $\left(\mathcal{L}_{\mathrm{I}}, \pi, \mathcal{R}_{\Lambda}\right) \in \mathrm{BLT}(S)$ then, by Theorem $5, \pi$ is idempotent-separating, so $\pi \subseteq \mu$. By Theorem $6,\left.\zeta\right|_{s} \subseteq \pi$.

$\Leftarrow:$ If $\pi \in\left[\left.\zeta\right|_{s}, \mu\right]$ then $\pi$ is necessarily idempotent-separating and therefore the triple $\left(\mathcal{L}_{1}, \pi, \mathcal{R}_{\Lambda}\right)$ is balanced. Now since, by the Corollary to Theorem $5,\left(\mathcal{L}_{\mathrm{l}}, \mu, \mathcal{R}_{\Lambda}\right) \in \operatorname{BLT}(S)$ the triple $\left(\mathcal{L}_{1}, \pi, \mathcal{R}_{\Lambda}\right)$ satisfies the conditions $[\alpha],[\gamma],[\delta],[\epsilon]$ the last two of which follow from the fact that $\pi \subseteq \mu$. Since $\left(\mathcal{L}_{\mathrm{I}},\left.\zeta\right|_{s}, \mathcal{R}_{\Lambda}\right) \in \operatorname{BLT}(S)$ and $\left.\zeta\right|_{s} \subseteq \pi$, the triple $\left(\mathcal{L}_{\mathrm{I}}, \pi, \mathcal{R}_{\Lambda}\right)$ also satisfies $[\beta]$. Hence $\left(\mathcal{L}_{\mathrm{I}}, \pi, \mathcal{R}_{\Lambda}\right) \in \operatorname{BLT}(S)$.

Corollary 1. Every $\pi \in\left[\left.\zeta\right|_{s^{\circ}}, \mu\right]$ is special.

Corollary 2. The ${ }^{\circ}$-congruences on $S$ that simultaneously extend $\mathcal{L}_{\mathrm{I}}$ and $\mathcal{R}_{\mathrm{\Lambda}}$ are precisely those of the form $\Psi\left(\mathcal{L}_{1}, \pi, \mathcal{R}_{\Lambda}\right)$ where $\pi \in\left[\left.\zeta\right|_{\varsigma^{\circ}}, \mu\right]$.

Example 2. Concerning the semigroup $Q$ of Example 1, we can describe the congruence $\zeta$ as follows. For every $X \in Q$ let $x_{11}$ be the entry in the $(1,1)$-position. Define a relation $\rho$ on $Q$ by

$$
(A, B) \in \rho \Leftrightarrow(\exists n \in \mathbb{Z}) \quad a_{11}=2^{n} b_{11} .
$$

It is easily seen that $\rho$ is a ${ }^{\circ}$-congruence on $Q$ that identifies $A$ and $A^{\circ \circ}$ for every $A \in Q$. Consequently, $\zeta \subseteq \rho$. Observe now that since the congruence $\zeta$ identifies the matrices

$$
\left[\begin{array}{ll}
x & x \\
x & x
\end{array}\right],\left[\begin{array}{ll}
x & 0 \\
0 & 0
\end{array}\right]
$$

we see, on pre-multiplying by $\left[\begin{array}{ll}1 & 1 \\ 1 & 1\end{array}\right]$, that $\zeta$ identifies the matrices

$$
\left[\begin{array}{ll}
2 x & 2 x \\
2 x & 2 x
\end{array}\right],\left[\begin{array}{ll}
x & 0 \\
0 & 0
\end{array}\right]
$$

and, by recursion, identifies the matrices

$$
\left[\begin{array}{ll}
2^{n} x & 2^{n} x \\
2^{n} x & 2^{n} x
\end{array}\right],\left[\begin{array}{ll}
x & 0 \\
0 & 0
\end{array}\right]
$$


If, therefore, $(A, B) \in \rho$ we have $a_{11}=2^{n} b_{11}$ where we can assume that the integer $n$ is non-negative, and consequently

$$
A \stackrel{\zeta}{\equiv}\left[\begin{array}{cc}
2^{n} b_{11} & 0 \\
0 & 0
\end{array}\right] \stackrel{\zeta}{\equiv}\left[\begin{array}{cc}
b_{11} & 0 \\
0 & 0
\end{array}\right] \stackrel{\zeta}{=} B
$$

Hence $\rho \subseteq \zeta$ and therefore $\zeta=\rho$.

We shall denote by $\omega_{s}$ the relation of equality on $S^{\circ}$. As the following result shows, the ${ }^{\circ}$-congruence $\zeta$ can be used to provide a measure of the distinction between quasiorthodox and orthodox.

Theorem 8. Let $S$ be a quasi-orthodox semigroup with an inverse transversal $S^{\circ}$. Then $S$ is orthodox if and only if $\left.\zeta\right|_{s}=\omega_{s}$.

Proof. $\Rightarrow$ : If $S$ is orthodox then we have the identity $(x y)^{\circ}=y^{\circ} x^{\circ}$ and so it follows by Theorem 3 that $\omega_{s^{\circ}} \in T$. Since $\left.\zeta\right|_{s^{\circ}}=\min T$ we deduce that $\left.\zeta\right|_{s^{\circ}}=\omega_{s^{\circ}}$.

$\Leftarrow$ : If $\left.\zeta\right|_{s^{\circ}}=\omega_{S^{\circ}}$ then $\Theta_{\omega_{s}} \in \overline{\operatorname{Con}} S$ and Theorem 3 gives the identity $(l i)^{\circ}=l^{\circ} i^{\circ}$. Thus $(\Lambda \mathrm{I})^{\circ} \subseteq E\left(S^{\circ}\right)$ and so $S^{\circ}$ is weakly multiplicative. Consequently, $S$ is orthodox.

Corollary. If $S$ is orthodox then $\left(\mathcal{L}_{\mathrm{I}}, \pi, \mathcal{R}_{\Lambda}\right) \in \operatorname{BLT}(S)$ if and only if $\pi$ is idempotentseparating.

Proof. This follows by Corollary 1 of Theorem 7.

\section{REFERENCES}

1. T. S. Blyth and M. H. Almeida Santos, A simplistic approach to inverse transversals, Proc. Edinburgh Math. Soc. 39 (1996), 57-69.

2. T. S. BLyth and M. H. Almeida SAntos, Congruences associated with inverse transversals, Collectanea Mathematica, memorial volume for Paul Dubreil, 46 (1995), 35-48.

3. T. E. HaLL, Congruences and Green's relations on regular semigroups, Glasgow Math. J. 13 (1972), 167-175.

4. D. B. MCAlister and R. MCFADDEN, Semigroups with inverse transversals as matrix semigroups, Quart. J. Math. Oxford 25 (1984), 455-474.

5. Tatsuhixo Sarto, Construction of regular semigroups with inverse transversals, Proc. Edinburgh Math. Soc. 32 (1989), 41-51.

6. TAtsuhiko Salto, Quasi-orthodox semigroups with inverse transversals, Semigroups Forum 36 (1987), 47-54.

7. XILIN TANG, Regular semigroups with inverse transversals, preprint.

8. M. Yamada, Structure of quasi-orthodox semigroups, Mem. Fac. Sc., Shimane Univ. 14 (1980), 1-18.

Mathematical InSTITUTE

UNIVERSITY OF ST ANDREWS

SCOTLAND
Departamento de Matemática

F.C.T.

UNiversidade Nova de Lisboa

PORTUGal 